Pacific Journal of Mathematics

ESTIMATES OF POSITIVE CONTRACTIONS

ESTImATES OF POSiTIVE CONTRCTONS 


\title{
ESTIMATES OF POSITIVE CONTRACTIONS
}

\author{
R. V. Chacon and S. A. McGrath
}

The purpose of this paper is to obtain an $L_{p}$ estimate for the supremum of the Cesàro averages of a certain class of positive contractions of $L_{p}$. Let $(X, \mathscr{F}, \mu)$ be a measure space, and let $T$ be a linear operator mapping $L_{p}(X, \mathscr{F}, \mu)$ into itself for $p$ fixed, $1<p<+\infty$. If there is a constant $c>0$ such that for each $f \in L_{p}(X, \mathscr{F}, \mu)$,

$$
\begin{gathered}
\int \sup _{n}\left|f,(f+T f) / 2, \cdots,\left(f+T f+\cdots+T^{n} f\right) / n+1\right|^{p} d \mu \\
\leqq c^{p} \int|f|^{p} d \mu,
\end{gathered}
$$

then we say that $T$ admits of a dominated estimate with constant $c$. In an effort to unify certain results due to $A$. IonescuTulcea and to $E$. Stein, a somewhat more general form of the following theorem was obtained earlier: If $T$ is a positive contraction, and if there exists an $h>0$ a.e., $h \in L_{p}(X, \mathscr{F}, \mu)$ and $T h=h$, then $T$ admits of a dominated estimate with constant $p / p-1$. In the present paper, we have extended the theorem, obtaining a slightly more general form of the following: If $T$ is a positive contraction and if for each positive integer $n$ there exists an $h_{n}>0$ a.e., $h_{n} \in L_{p}(X, \mathscr{F}, \mu)$ and $\left\|h_{n}\right\|=\left\|T^{n} h_{n}\right\|$, then $T$ admits of a dominated estimate with constant $p / p-1$.

This result is more widely applicable more directly than the previous theorem, but is not the most general result one might conjecture, that positive contractions admit of a dominated estimate with no further assumptions. In this direction, we have obtained several equivalent formulations of the problem which may help to lead to an answer. In any case, it remains an open problem whether or not positive contractions of $L_{p}(X, \mathscr{F}, \mu), 1<p<+\infty$, admit of a dominated estimate without the assumption of additional conditions.

2. Main results. Let $\left(X_{1}, \mathscr{F}_{1}, \mu_{1}\right)$ and $\left(X_{2}, \mathscr{F}_{2}, \mu_{2}\right)$ be two measure spaces and let $T$ be a linear operator mapping $L_{p}\left(X_{1}, \mathscr{F}_{1}, \mu_{1}\right)$ into $L_{p}\left(X_{2}, \mathscr{F}_{2}, \mu_{2}\right), p$ fixed, $1 \leqq p \leqq+\infty$. We say that $T$ is a contraction if its norm is less than or equal to one. We say that $T$ is positive if it maps nonnegative functions to nonnegative functions. We shall omit the phrase almost everywhere, it being understood where applicable.

Definition 2.1. The range set of $T, R(T)$, is the support of $T f$, 
where $f \in L_{p}\left(X_{1}, \mathscr{F}_{1}, \mu_{1}\right)$ and $f>0$ (it is clear that $R(T) \in \mathscr{F}_{2}$ and that it is independent of the particular $f>0$ chosen).

Lemma 2.1. Let $T$ be a linear operator of $L_{p}\left(X_{1}, \mathscr{F}_{1}, \mu_{1}\right)$ into $L_{p}\left(X_{2}, \mathscr{F}_{2}, \mu_{2}\right), p$ fixed, $1<p<+\infty$, and let

$$
h_{1} \in L_{p}\left(X_{1}, \mathscr{F}_{1}, \mu_{1}\right), h_{2} \in L_{p}\left(X_{2}, \mathscr{F}_{2}, \mu_{2}\right)
$$

be nonnegative functions, $h_{1}>0$ on $R\left(T^{*}\right)$ and $h_{2}>0$ on $R(T)$. Let $P$ be the linear operator of $L_{p}\left(X_{1}, \mathscr{F}_{1}, m_{1}\right)$ into $L_{p}\left(X_{2}, \mathscr{F}_{2}, m_{2}\right)$ defined by

$$
P(f)=\left\{\begin{array}{l}
T\left(f h_{1}\right) / h_{2} \text { on } R(T), \\
0 \text { otherwise }
\end{array}\right.
$$

where the measures $m_{1}$ and $m_{2}$ are obtained by setting

$$
m_{1}\left(A_{1}\right)=\int_{A_{1}} h_{1}^{p} d \mu_{1}, A_{1} \in \mathscr{F}_{1} \quad \text { and } \quad m_{2}\left(A_{2}\right)=\int_{A_{2}} h_{2}^{p} d \mu_{2}, A_{2} \in \mathscr{F}_{2} .
$$

We have then that

( i ) $\|P\| \leqq\|T\|$

and that

(ii) $P^{*} g=\left\{\begin{array}{l}T^{*}\left(g h_{2}^{p-1}\right) / h_{1}^{p-1} \text { on } R\left(T^{*}\right), \\ 0 \text { otherwise. }\end{array}\right.$

Furthermore, if $T$ is a positive contraction, $\left\|h_{1}\right\|=\left\|h_{2}\right\|$, and $T h_{1}=h_{2}$ on $R(T)$, then we have

(iii) $P^{*} 1=1$ (or equivalently by (ii), $T^{*} h_{2}^{p-1}=h_{1}^{p-1}$ ) on $R\left(T^{*}\right)$.

Proof. We adapt the proof of Lemma 3.1 of [1]. To see that (i) holds,

$$
\begin{aligned}
& \int|P f|^{p} d m_{2}=\int\left|T\left(f h_{1}\right) / h_{2}\right|^{p} h_{2}^{p} d \mu_{2} \\
& \quad=\int\left|T\left(f h_{1}\right)\right|^{p} d \mu_{2} \leqq\|T\|^{p} \int\left|f h_{1}\right|^{p} d \mu_{1} \\
& \quad=\|T\|^{p} \int|f|^{p} d m_{1},
\end{aligned}
$$

so that $\|P\| \leqq\|T\|$. To see that (ii) holds,

$$
\int g P(f) d m_{2}=\int g\left[T\left(f h_{1}\right) / h_{2}\right] h_{2}^{p} d \mu_{2}=\int T^{*}\left(g h_{2}^{p-1}\right) / h_{1}^{p-1} f d m_{1} .
$$

Finally, to see that (iii) holds, define $\alpha(x)$ by setting $P^{*} 1=1+\alpha(x)$. Then, with $q=p / p-1$, we have

$$
\int 1 d m_{2}=\int 1^{q} d m_{2} \geqq \int\left(P^{*} 1\right)^{q} d m_{1}=\int(1+\alpha)^{q} d m_{1}
$$


since $\left\|P^{*}\right\| \leqq 1$ by (i). Also

$$
\int(1+\alpha)^{q} d m_{1} \geqq \int 1 d m_{1}+q \int \alpha d m_{1}
$$

with strict inequality if $\alpha \neq 0$ on a set of positive measure, since $(1+\alpha(x))^{q}>1+q \alpha(x)$ for each $x$ such that $\alpha(x) \neq 0$. Further, if $T h_{1}=h_{2}$ on $R(T), P(1)=1$ on $R(T)=R(P)$ and

$$
\int P^{*}(1) d m_{1}=\int 1 P(1) d m_{2}=\int 1 d m_{2}=\int 1 d m_{1}+\int \alpha d m_{1} .
$$

If, in addition, we have that $\left\|h_{1}\right\|=\left\|h_{2}\right\|$, then $\int d m_{2}=\int d m_{1}$, and this equation then implies $\int \alpha d m_{1}=0$. This is incompatible with the previous inequalities (if, as we have, $\left\|h_{1}\right\|=\left\|h_{2}\right\|$ ) unless $\alpha(x)=0$ on $R\left(T^{*}\right)$ and $P^{*} 1=1$ on $R\left(T^{*}\right)$.

REMARK 2.1. Lemma 2.1 includes Lemma 3.1 of [1] as we can see by taking $(X, \mathscr{F}, \mu)=\left(X_{1}, \mathscr{F}_{1}, \mu_{1}\right)=\left(X_{2}, \mathscr{F}_{2}, \mu_{2}\right)$. That $T h_{1}=h_{2}$, $\left\|h_{1}\right\|=\left\|h_{2}\right\|$ is certainly satisfied if there exists $h>0$ such that $T h=h$ by taking $h_{1}=h_{2}=h$. Further, the slight generalization of Lemma 3.1 of [1] which is the one-dimensional version of this result implies the two-dimensional (by taking

$$
(X, \mathscr{F}, \mu)=\left(X_{1}, \mathscr{F}_{1}, \mu_{1}\right) \oplus\left(X_{2}, \mathscr{F}_{2}, \mu_{2}\right),
$$

$\left.T=T_{1} \oplus 0\right)$. We have stated in this form primarily because of the application we make of it.

LEMma 2.2. Let $T$ be a positive contraction of $L_{p}\left(X_{1}, \mathscr{F}_{1}, \mu_{1}\right)$ into $L_{p}\left(X_{2}, \mathscr{F}_{2}, \mu_{2}\right), p$ fixed, $1<p<+\infty$, and let $h_{1} \in L_{p}\left(X_{1}, \mathscr{F}_{1}, \mu_{1}\right)$, $h_{2} \in L_{p}\left(X_{2}, \mathscr{F}_{2}, \mu_{2}\right)$ be nonnegative functions, $h_{1}>0$ on $R\left(T^{*}\right), h_{2}>0$ on $R(T)$ and such that $T h_{1} \leqq h_{2}$ on $R(T)$ and $T^{*} h_{2}^{p-1} \leqq h_{1}^{p-1}$ on $R\left(T^{*}\right)$. Let $P$ be the positive linear operator of $L_{p}\left(X_{1}, \mathscr{F}_{1}, m_{1}\right)$ into $L_{p}\left(X_{2}, \mathscr{F}_{2}, m_{2}\right)$ defined by

$$
P f=\left\{\begin{array}{l}
T\left(f h_{1}\right) / h_{2} \text { on } R(T), \\
0 \text { otherwise }
\end{array}\right.
$$

where the measures $m_{1}$ and $m_{2}$ are obtained by setting

$$
m_{1}\left(A_{1}\right)=\int_{A_{1}} h_{1}^{p} d \mu_{1}, A_{1} \in \mathscr{F}_{1} \quad \text { and } \quad m_{2}\left(A_{2}\right)=\int_{A_{2}} h_{2}^{p} d \mu_{2},
$$

$A_{2} \in \mathscr{F}$ (as in Lemma 2.1). Then $P$ is a positive contraction of $L_{1}\left(X_{1}, \mathscr{F}_{1}, m_{1}\right)$ into $L_{1}\left(X_{2}, \mathscr{F}_{2}, m_{2}\right)$ and also of $L_{\infty}\left(X_{1}, \mathscr{F}_{1}, m_{1}\right)$ into $L_{\infty}\left(X_{2}, \mathscr{F}_{2}, m_{2}\right)$. 
Proof. It follows by part (i) of Lemma 2.1 that $P$ is a positive contraction of $L_{p}\left(X_{1}, \mathscr{F}_{1}, m_{1}\right)$ into $L_{p}\left(X_{2}, \mathscr{F}_{2}, m_{2}\right)$. It is clear from the definition that $P 1 \leqq 1$, implying that $P$ is a positive contraction of $L_{\infty}\left(X_{1}, \mathscr{F}_{1}, m_{1}\right)$.

We have that $P^{*}$ is a positive contraction of $L_{q}\left(X_{2}, \mathscr{F}_{2}, m_{2}\right)$ into $L_{q}\left(X_{1}, \mathscr{F}_{1}, m_{1}\right)(q=p / p-1)$ and by part (ii) of Lemma 2.1 that $P^{*} 1 \leqq 1$ and therefore that $P^{*}$ is a positive contraction of $L_{\infty}\left(X_{2}, \mathscr{F}_{2}, m_{2}\right)$ into $L_{\infty}\left(X_{1}, \mathscr{F}_{1}, m_{1}\right)$. An application of the Riesz convexity theorem then implies that $P$ is a contraction of $L_{1}\left(X_{1}, \mathscr{F}_{1}, m_{1}\right)$ into $L_{1}\left(X_{2}, \mathscr{F}_{2}, m_{2}\right)$.

We next state the dominated ergodic theorem (see [1] for an outline of its proof with the constant given here).

Lemma 2.3. (Dominated ergodic theorem.) Let $T$ be a contraction of $L_{1}(X, \mathscr{F}, \mu)$ into itself and of $L_{\infty}(X, \mathscr{F}, \mu)$ into itself. Then $T$ admits of a dominated estimate with constant $p / p-1$.

THEOREM 2.1. Let $\left(X_{i}, \mathscr{F}_{i}, \mu_{i}\right), i=1,2, \cdots, n$ be measure spaces, and let $T_{i}$ be a positive contraction of

$$
L_{p}\left(X_{i}, \mathscr{F}_{i}, \mu_{i}\right) \text { into } L_{p}\left(X_{i+1}, \mathscr{F}_{i+1}, \mu_{i+1}\right)
$$

for $i=1,2, \cdots, n-1$. Let $h_{i} \in L_{p}\left(X_{i}, \mathscr{F}_{i}, \mu_{i}\right)$ be nonnegative functions, $i=1,2, \cdots, n$ such that

(i) $h_{i}>0$ on $R\left(T_{i}^{*}\right), i=1, \cdots, n-1, h_{i}>0$ on $R\left(T_{i-1}\right), i=$ $2, \cdots, n$,

(ii) $T_{i} h_{i} \leqq h_{i+1}$, on $R\left(T_{i}\right)$,

(iii) $T_{i}^{*} h_{i+1}^{p-1} \leqq h_{i}^{p-1}$, on $R\left(T_{i}^{*}\right)$,

for $i=1,2, \cdots, n-1$. Then we have

$$
\begin{aligned}
& \sum_{i=1}^{n} \int \sup \mid f_{i},\left(f_{i}+T_{i-1} f_{i-1}\right) / 2, \cdots, \\
& \left(f_{i}+T_{i-1} f_{i-1}+\cdots+T_{i-1} T_{i-2} \cdots T_{1} f_{1}\right) /\left.i\right|^{p} d \mu_{i} \\
& \leqq \\
& \leqq \\
&
\end{aligned}
$$

where $f_{i} \in L_{p}\left(X_{i}, \mathscr{F}_{i}, \mu_{i}\right)$.

Proof. Let $(X, \mathscr{F}, \mu)=\bigoplus_{i=1}^{n}\left(X_{i}, \mathscr{F}_{i}, \mu_{i}\right)$. A function $f$ on $X$ can be written as an $n$-tuple of functions, $f=\left(f_{1}, \cdots, f_{n}\right)$ where $f_{i}$ is a function on $X_{i}, i=1,2, \cdots, n$. The norm of $f$ is given by $\|f\|=$ $\left(\sum_{i=1}^{n}\left\|f_{i}\right\|^{p}\right)^{1 / p}$.

We define a positive contraction $T$ of $L_{p}(X, \mathscr{F}, \mu)$ into $L_{p}(X, \mathscr{F}, \mu)$ by setting

$$
T\left(f_{1}, \cdots, f_{n}\right)=\left(0, T_{1} f_{1}, \cdots, T_{n-1} f_{n-1}\right) .
$$


That $T$ is a contraction follows from

$$
\left\|T\left(f_{1}, \cdots, f_{n}\right)\right\|^{p}=\sum_{i=1}^{n-1}\left\|T_{i} f_{i}\right\|^{p} \leqq \sum_{i=1}^{n-1}\left\|f_{i}\right\|^{p} \leqq\|f\|^{p} .
$$

We next calculate $T^{*} g$. Let

$$
f \in L_{p}(X, \mathscr{F}, \ell) \text { and let } T^{*}(g)=\left(g_{1}^{*}, \cdots, g_{n}^{*}\right) .
$$

Then

$$
\begin{aligned}
\int T^{*}(g) f d \mu & =\int\left(g_{1}^{*}, \cdots, g_{n}^{*}\right)\left(f_{1}, \cdots, f_{n}\right) d \mu \\
& =\sum_{i=1}^{n-1} \int g_{i}^{*} f_{i} d \mu_{i}=\int T(f) g=\sum_{i=1}^{n-1} \int\left(T_{i} f_{i}\right) g_{i+1} d \mu_{i+1} \\
& =\sum_{i=1}^{n-1} \int T_{i}^{*}\left(g_{i+1}\right) f_{i} d \mu_{i} .
\end{aligned}
$$

Since $f$ is arbitrary, it follows that $T^{*}(g)=\left(T_{1}^{*} g_{2}, \cdots, T_{n-1}^{*} g_{n}, 0\right)$.

We may apply Lemma 2.2 to each pair

$$
L_{p}\left(X_{i}, \mathscr{F}_{i}, \mu_{i}\right), L_{p}\left(X_{i+1}, \mathscr{F}_{i+1}, \mu_{i+1}\right)
$$

(or to $(X, \mathscr{F}, \mu)$ and $\left(h_{1}, h_{2}, \cdots, h_{n}\right)$ each taken twice) to obtain that the operator

$$
\operatorname{Pr}=\left(0, P_{1} r_{1}, \cdots, P_{n-1} r_{n-1}\right)
$$

of

$$
L_{p}(X, \mathscr{F}, m)=\bigoplus_{i=1}^{n}\left(X_{i}, \mathscr{F}_{i}, m_{i}\right)
$$

into itself is a contraction of $L_{1}(X, \mathscr{F} ; m)$ into $L_{1}(X, \mathscr{F}, m)$ and also of $L_{\infty}(X, \mathscr{F}, m)$ into $L_{\infty}(X, \mathscr{F}, m)$, where

$$
P_{i} r_{i}=\left\{\begin{array}{l}
T_{i}\left(r_{i} h_{i}\right) / h_{i+1} \text { on } R\left(T_{i}\right), \\
0 \text { otherwise }
\end{array}\right.
$$

and

$$
m_{i}\left(A_{i}\right)=\int_{A_{i}} h_{i}^{p} d \mu_{i}, A_{i} \in \mathscr{F}_{i} .
$$

An application of Lemma 2.3 then yields

$$
\begin{aligned}
& \int \sup \left|r, r+\operatorname{Pr} / 2, \cdots,\left(r+\cdots+P^{k-1} r\right) / k, \cdots\right|^{p} d m \\
& \quad \leqq(p / p-1)^{p} \int|r|^{p} d m
\end{aligned}
$$

Now 


$$
\begin{aligned}
& \left(r+\operatorname{Pr}+\cdots P^{k-1} r\right) / k \\
& =\left\{\begin{array}{l}
\left(r_{1} / k,\left(r_{2}+P_{1} r_{1}\right) / k, \cdots,\right. \\
\left.\quad\left(r_{n}+P_{n-1} r_{n-1}+\cdots+P_{n-1} \cdots P_{n-k+1} r_{n-k+1}\right) / k\right) \\
\quad \text { for } k<n-1, \\
\left(r_{1} / k,\left(r_{2}+P_{1} r_{1}\right) / k, \cdots,\right. \\
\left.\quad\left(r_{n}+P_{n-1} r_{n-1}+\cdots+P_{n-1} \cdots P_{1} r_{1}\right) / k\right) \\
\text { for } k \geqq n-1,
\end{array}\right.
\end{aligned}
$$

and, therefore, Lemma 2.3 implies that

$$
\begin{aligned}
& \sum_{i=1}^{n} \int \sup \mid r_{i},\left(r_{i}+P_{i-1} r_{i-1}\right) / 2, \cdots, \\
& \left(r_{i}+P_{i-1} r_{i-1}+\cdots+P_{i-1} \cdots P_{1} r_{1}\right) /\left.i\right|^{p} d m_{i} \\
& \leqq(p / p-1)^{p} \sum_{i=1}^{n} \int\left|r_{i}\right|^{p} d m_{i} .
\end{aligned}
$$

The theorem follows from this on setting

$$
r_{i}=f_{i} / h_{i}, d m_{i}=h_{i}^{p} d \mu_{i}
$$

and

$$
P_{i-1} \cdots P_{i-j} r_{i-j}=\left(T_{i-1} \cdots T_{i-j} r_{i-j} h_{i-j}\right) / h_{i-j}
$$

Lemma 2.4. Let $\left(X_{1}, \mathscr{F}_{1}, \mu_{1}\right)$ and $\left(X_{2}, \mathscr{F}_{2}, \mu_{2}\right)$ be measure spaces, and let $T$ be a positive contraction of $L_{p}\left(X_{1}, \mathscr{F}_{1}, \mu_{1}\right)$ into $L_{p}\left(X_{2}, \mathscr{F}_{2}, \mu_{2}\right)$. Let $f \in L_{p}\left(X_{1}, \mathscr{F}_{1}, \mu_{1}\right)$ and $g \in L_{p}\left(X_{1}, \mathscr{F}_{1}, \mu_{1}\right)$ be nonnegative functions such that $f$ vanishes outside the support of $g$. Then $T f$ vanishes outside the support of $T g$.

Proof. Follows at once by contradiction.

LEMmA 2.5. Let $\left(X_{1}, \mathscr{F}_{1}, \mu_{1}\right)$ and $\left(X_{2}, \mathscr{F}_{2}, \mu_{2}\right)$ be measure spaces, and let $T$ be a positive contraction of $L_{p}\left(X_{1}, \mathscr{F}_{1}, \mu_{1}\right)$ into $L_{p}\left(X_{2}, \mathscr{F}_{2}, \mu_{2}\right)$. Let $f, g \in L_{p}\left(X_{1}, \mathscr{F}_{1}, \mu_{1}\right)$ be nonnegative and $g$ such that $\|g\|=\|T g\|$. If $f$ vanishes on the support of $g$, then $T f$ vanishes on the support of $T g$.

Proof. Let $E$ denote the support of $g$ and $F$ the support of $T g$. Suppose $T f$ does not vanish of $F$. Then we can find a subset $A$ of $F \cap\{x: T f>0\}$ having positive measure and a positive number $\alpha$ such that $\alpha T g<T f$ on $A$. We have for any $\beta>0$

$$
\begin{aligned}
\beta^{p}\|g\|^{p}+\|f\|^{p}=\|\beta g+f\|^{p} \geqq\|\beta T g+T f\|^{p} \\
>\left\|(\beta T g+\alpha T g) \psi_{A}\right\|^{p}+\left\|\beta T g \psi_{X-A}\right\|^{p} \\
=(\beta+\alpha)^{p}\left\|T g \psi_{A}\right\|^{p}+\left\|\beta T g \psi_{X-A}\right\|^{p} .
\end{aligned}
$$


Thus, since $\|T g\|=\|g\|$,

$$
\beta^{p}\left\|(T g) \psi_{A}\right\|^{p}+\|f\|^{p}>(\alpha+\beta)^{p}\left\|(T g) \psi_{A}\right\|^{p} .
$$

For $\beta$ sufficiently large,

$$
(\alpha+\beta)^{p}=\beta^{p}\left(1+\frac{\alpha}{\beta}\right)^{p}>\beta^{p}\left(1+p \frac{\alpha}{\beta}\right)=\beta^{p}+p \beta^{p-1} \alpha
$$

implying

$$
\|f\|^{p}>p \beta^{p-1} \alpha\left\|T g \cdot \psi_{A}\right\|^{p}
$$

for $\beta$ sufficiently large, which is impossible since $\|f\|^{p}<+\infty$.

Lemma 2.6. Let $(X, \mathscr{F}, \mu)$ be a measure space, and let $T$ be a positive contraction of $L_{p}(X, \mathscr{F}, \mu)$. Suppose that for some $n$, there exist $n$ positive functions $h_{1 n}, \cdots, h_{n n}$ in $L_{p}(X, \mathscr{F}, \mu)$ such that

$$
\begin{aligned}
& T h_{k n} \leqq h_{k+1 n} \text { on } R(T) \\
& T^{*} h_{k+1 n}^{p-1} \leqq h_{k n}^{p-1} \text { on } R\left(T^{*}\right)
\end{aligned}
$$

for $k=1, \cdots, n-1$. Then, for $f \in L_{p}(X, \mathscr{F}, \mu)$, we have

$$
\begin{aligned}
& \frac{1}{n} \sum_{i=1}^{n} \int \sup \left|f,(f+T f) / 2, \cdots,\left(f+\cdots+T^{i-1} f\right) / i\right|^{p} d \mu \\
& \quad \leqq(p / p-1)^{p} \int|f|^{p} d \mu .
\end{aligned}
$$

Proof. The lemma follows at once from Theorem 2.1 if we let $\left(X_{i}, \mathscr{F}_{i}, \mu_{i}\right)=(X, \mathscr{F}, \mu), T_{i}=T$, and $f_{i}=f, i=1, \cdots, n$.

Lemma 2.7. Let $(X, \mathscr{F}, \mu)$ be a measure space, and let $T$ be a positive contraction of $L_{p}(X, \mathscr{F}, \mu)$. Suppose that for some $n \geqq 1$, there exists a positive function $h_{n} \in L_{p}(X, \mathscr{F}, \mu)$ such that $\left\|T^{n} h_{n}\right\|=$ $\left\|h_{n}\right\|$. Then there exist $n+1$ positive functions $h_{1 n}, \cdots, h_{n+1 n}$ in $L_{p}(X, \mathscr{F}, \mu)$ such that

$$
\begin{aligned}
& T h_{k n}=h_{k+1 n} \text { on } R(T), \\
& T^{*} h_{k+1 n}^{p-1}=h_{k n}^{p-1} \text { on } R\left(T^{*}\right),
\end{aligned}
$$

for $k=1, \cdots, n$.

Proof. We let $h_{1 n}=h_{n}$, and $h_{k+1 n}=T h_{k n}+\psi_{c R(T)} h_{k n}, k=1, \cdots, n$. It is clear from the definition that the functions $h_{1 n}, \cdots, h_{n+1 n}$ are positive and that $T h_{k n}=h_{k+1 n}$ on $R(T), k=1, \cdots, n$. It also follows easily from the definition of $T^{*}$ that 


$$
T^{*} h_{k+1 n}^{p-1}=T^{*}\left(T h_{k n}\right)^{p-1}, k=1, \cdots, n .
$$

If we show that $\left\|h_{k n}\right\|=\left\|T h_{k n}\right\|, k=1, \cdots, n$, then we would have also shown that $T^{*} h_{k+1 n}^{p-1}=h_{k n}^{p-1}$ on $R\left(T^{*}\right), k=1, \cdots, n$, by part (iii) of Lemma 2.1, since $T^{*} h_{k+1 n}^{p-1}=T^{*}\left(T h_{k n}\right)^{p-1}, k=1, \cdots, n$ on $R\left(T^{*}\right)$. To see that $\left\|h_{k n}\right\|=\left\|T h_{k n}\right\|, k=1, \cdots, n$, we'll prove a little more, that $\left\|h_{k n}\right\|=\left\|T^{i} h_{k n}\right\|, i=1, \cdots, n-k+1, k=1, \cdots, n$. We proceed by induction. That $\left\|h_{1 n}\right\|=\left\|T^{i} h_{1 n}\right\|, i=1, \cdots, n$ holds by hypothesis. Next, we suppose $\left\|h_{k(0) n}\right\|=\left\|T^{i} h_{k(0) n}\right\|, i=1, \cdots, n-k(0)+1$ for $k(0)<n$. We write $h_{k(0)+1 n}=T h_{k(0) n}+\psi_{c R(T)} h_{k(0) n}$, and since the supports of $T h_{k(0) n}$ and of $\psi_{c R(T)} h_{k(0) n}$ are disjoint, and since by the induction hypothesis $\left\|T h_{k(0) n}\right\|=\left\|T^{i} h_{k(0) n}\right\|, i=1, \cdots, n-k(0)+1$ it follows by Lemma 2.5 that $T^{i} T h_{k(0) n}$ and $T \psi_{c R(T)} h_{k(0) n}$ have disjoint support, and hence that $T^{i} \psi_{R(T)} h_{k(0) n}$ and $T_{c R(T)}^{i} h_{k(0) n}$ have disjoint support, $i=$ $1, \cdots, n-k(0)$ (since the supports of $T h_{k(0) n}$ and of $\psi_{R(T)} h_{k(0) n}$ are the same). That $T^{i} \psi_{R(T)}, h_{k(0) n}$ and $T^{i} \psi_{c R(T)} h_{k(0) n}$ have disjoint support, $i=$ $1, \cdots, n-k(0)$ and the induction hypothesis imply that

$$
\left\|T^{i} \psi_{c R(T)} h_{k(0) n}\right\|=\left\|\psi_{c R(T)} h_{k(0) n}\right\|, i=1, \cdots, n-k(0) .
$$

That $T^{i} T h_{k(0) n}$ and $T^{i} \psi_{c R(T)} h_{k(0) n}$ have disjoint support, $i=1, \cdots, n-k(0)$, implies that

$$
\begin{aligned}
\left\|T^{i} h_{k(0)+1 n}\right\| & =\left\|T^{i} T h_{k(0) n}\right\|+\left\|T^{i} \psi_{c R(T)} h_{k(0) n}\right\| \\
& =\left\|T^{i} T h_{k(0) n}\right\|+\left\|\psi_{c R(T)} h_{k(0) n}\right\|,
\end{aligned}
$$

$i=1, \cdots, n-k(0)$, since

$$
\left\|T^{i} \psi_{c R(T)} h_{k(0) n}\right\|=\left\|\psi_{c R(T)} h_{k(0) n}\right\|, i=1, \cdots, n-k(0) .
$$

This equation implies by the induction hypothesis, that

$$
\begin{aligned}
\left\|T^{i} h_{k(0)+1 n}\right\| & =\left\|T h_{k(0) n}\right\|+\left\|\psi_{c R(T)} h_{k(0) n}\right\|_{1} \\
& =\left\|h_{k(0)+1 n}\right\|,
\end{aligned}
$$

$i=1, \cdots, n-k(0)$, from which the lemma follows, since $n-k(0)=$ $n-(k(0)+1)+1$.

Lemma 2.8. Let $(X, \mathscr{F}, \mu)$ be a measure space, and let $T$ be a positive contraction of $L_{p}(X, \mathscr{F}, \mu)$. Suppose that for some $n$ there exists a positive function $h_{n}$ such that $\left\|T^{n} h_{n}\right\|=\left\|h_{n}\right\|$. Then, for $f \in L_{p}(X, \mathscr{F}, \mu)$ we have

$$
\begin{aligned}
& \frac{1}{n+1} \sum_{i=1}^{n+1} \int \sup \left|f,(f+T f) / 2, \cdots,\left(f+\cdots+T^{\imath-1} f\right) / 2\right|^{p} d \mu \\
& \quad \leqq(p / p-1)^{p} \int|f|^{p} d \mu .
\end{aligned}
$$


THeorem 2.2. Let $(X, \mathscr{F}, \mu)$ be a measure space, and let $T$ be a positive contraction of $L_{p}(X, \mathscr{F}, \mu)$. Suppose that for each $n$ there exists a positive function $h_{n}$ such that $\left\|T^{n} h_{n}\right\|=\left\|h_{n}\right\|$. Then $T$ admits of a dominated estimate with constant $p / p-1$.

Proof. Follows at once from Lemma 2.8.

3. Equivalent formulations. In this section, we obtain various sets of necessary and sufficient conditions for a positive contraction of $L_{1}$ to admit of a dominated estimate, and same related results.

Definition 3.1. Let $(X, \mathscr{F}, \mu)$ be a measure space. For

$$
1 \leqq p(1) \leqq p(2) \leqq+\infty,
$$

we define the class of positive contractions $\mathscr{A}\left(p_{1}, p_{2}\right)$ setting $\mathscr{A}\left(p_{1}, p_{2}\right)=$ $\left\{T: T\right.$ is a positive contraction of $\left.L_{p}(X, \mathscr{F}, \mu), p(1) \leqq p \leqq p(2)\right\}$.

THEOREM 3.1. If $1<p(0)<+\infty$, then the following are equivalent:

(i ) If $T \in \mathscr{A}(1, p(0))$, then $T$ (regarded as a contraction of $\left.L_{p(0)}(X, \mathscr{F}, \mu)\right)$ admits of a dominate estimate with constant $c(p(0))$.

(ii) If $T \in \mathscr{A}(p(0), p(0))$, then $T$ admits of a dominated estimate with constant $c(p(0))$.

(iii) If $T \in \mathscr{A}(p(0),+\infty)$, then $T$ (regarded as a contraction of $\left.L_{p(0)}(X, \mathscr{F}, \mu)\right)$ admits of a dominated estimate with constant $c(p(0))$.

Proof. Part (a), (i) implies (ii). Let $T \in \mathscr{A}(p(0), p(0))$, and suppose $\|T\|=\delta<1$. Then $\left\|T^{*}\right\|=\delta<1$ and there exists a positive function $\hbar \in L_{q(0)}(X, \mathscr{F}, \mu)$, with $q(0)=p(0) / p(0)-1$, such that $T^{*} \hbar \leqq \hbar$ (to see this, let $f \in L_{q(0)}(X, \mathscr{F}, \mu)$ be an arbitrary positive function, and let $\left.\hbar=\sum_{k=0}^{\infty} T^{*} f\right)$. If $h=(h)^{1 / p(0)-1}$ then

$$
h \in L_{p(0)}(X, \mathscr{F}, \mu) \text { and } T^{*} h^{p(0)-1} \leqq h^{p(0)-1} .
$$

We may then define a transformation $P$ of $L_{p(0)}(X, \mathscr{F}, m)$ by setting, for $f \in L_{p(0)}(X, \mathscr{F}, m)$

$$
P(f)=T(f \cdot h) / h
$$

where $m$ is the measure given by $m(A)=\int_{A} h^{p(0)} d \mu$.

It follows by part (i) of Lemma 2.1 (with $h_{1}=h_{2}=h$, and

$$
\left.\left(X_{1}, \mathscr{F}_{1}, \mu_{1}\right)=\left(X_{2}, \mathscr{F}_{2}, \mu_{2}\right)=(X, \mathscr{F}, \mu)\right)
$$

that $\|P\|_{p(0)} \leqq\|T\|_{p(0)}$. Part (ii) of Lemma 2.1 implies that $P^{*} 1 \leqq 1$, 
and since $P$ is positive, it follows by the Riesz convexity theorem that $P \in \mathscr{A}(1, p(0))$ (with respect to the measure space $(X, \mathscr{F}, m)$ ). Since there is a set isomorphism of $(X, \mathscr{F}, m)$ into $(X, \mathscr{F}, \mu)$ (it may be necessary to choose $h$ with a sufficiently small norm), we have by assumption that $P$ admits of a dominated estimate with constant $c(p(0))$. Since we have that $P^{n} f=T^{n}(f h) / h$, and since $g \in L_{p(0)}(X, \mathscr{F}, \mu)$ implies $g / h \in L_{p(0)}(X, \mathscr{F}, m)$, we have that $T$ admits of a dominated estimate with constant $c(p(0))$ if $P$ does, and this finishes the proof of part (a) under the assumption that $\|T\|=\delta<1$.

Next, suppose $\|T\|=1$. Define $T_{\alpha}=\alpha T$ for $0<\alpha \leqq 1$. For $g \in L_{p(0)}(X, \mathscr{F}, \mu)$, let $g_{\alpha}^{*}$ denote $\sup _{n}\left|\left(g+\cdots+T_{\alpha}^{n} g\right) / n+1\right|$. If $g \geqq 0$, then $\left\|g_{\alpha}^{*}\right\|_{p(0)}$ converges monotonically to $\left\|g_{1}^{*}\right\|_{p^{(0)}}$, and the general case then follows from the special case, $\|T\|<1$, by the Lebesgue monotone convergence theorem.

Part (b), (iii) $\Rightarrow$ (ii). Let $T \in \mathscr{A}(p(0), p(0))$ and assume $\|T\|=$ $\delta<1$. Let $h$ be a positive function in $L_{p(0)}(X, \mathscr{F}, \mu)$ such that $T h \leqq h$. Define

$$
P f=T(f h) / h
$$

for $f \in L_{p(0)}(X, \mathscr{F}, m)$ where $m(A)=\int_{A} h^{p(0)} d \mu$. Then $P$ is a contraction of $L_{p(0)}(X, \mathscr{F}, m)$ since $\|P\|_{p(0)} \leqq\|T\|_{p(0)}$ by part (i) of Lemma 2.1. We also have $P(1) \leqq 1$, and hence $P \in \mathscr{A}(p(0),+\infty)$ (with respect to $(X, \mathscr{F}, m))$ again by the isomorphism result used in part (a) of the proof, we have that $P$ admits of a dominated estimate with constant $c(p(0))$, by assumption. In a similar way to that of part (a), we have that $T$ admits of a dominated estimate with constant $c(p(0))$ if $P$ does, and the general case $(\|T\| \leqq 1)$ follows from the special case by taking limits of $T_{\alpha}=\alpha T$ as in part (a).

Part (c), (ii) implies (i) and (iii). This case is trivial since

$$
\mathscr{A}(1, p(0)) \subset \mathscr{A}(p(0), p(0)) \text {, }
$$

and since $\mathscr{A}(p(0),+\infty) \subset \mathscr{A}(p(0), p(0))$.

THEOREM 3.2. If for each

$$
(X, \mathscr{F}, \mu), T \in \mathscr{A}(p(0), p(0)), 1<p(0)<+\infty,
$$

admits of a dominated estimate with constant $c(p(0), f, T,(X, \mathscr{F}, \mu))$, then there exists a constant $c(p(0)$ ), independent of $T$ and of $f$ (and of $(X, \mathscr{F}, \mu))$ such that each $T \in \mathscr{A}(p(0), p(0))$ will admit of a dominated estimate with constant $c(p(0))$.

Proof. Note that we have used an obvious extension of a dominated estimate with constant dependent on the particular function 
used. Suppose to the contrary. Then there exists a sequence of spaces $\left(X_{n}, \mathscr{F}_{n}, \mu_{n}\right)$ and a sequence $\left\{f_{n}\right\}$ of positive functions with $f_{n}$ in $L_{p(0)}\left(X_{n}, \mathscr{F}_{n}, \mu_{n}\right)$ such that $\left\|f_{n}\right\|^{p(0)}=1 / 2^{n}, n=1,2, \cdots$, and a sequence $\left\{T_{n}\right\}$ of positive contractions in $\mathscr{A}(p(0), p(0))$ such that $\left\|T_{n}\right\| \leqq 1$, and such that

$$
\begin{aligned}
& \int \sup _{j}\left|f_{n},\left(f_{n}+T_{n} f_{n}\right) / 2, \cdots,\left(f_{n}+\cdots+T_{n}^{j-1} f_{n}\right) / j\right|^{p(0)} d u_{n} \\
& \quad \geqq 4^{n} \int\left|f_{n}\right|^{p(0)} d \mu_{n} .
\end{aligned}
$$

Let $(Y, \mathscr{B}, m)=\bigoplus_{n=1}^{\infty}\left(X_{n}, \mathscr{F}_{n}, \mu_{n}\right)$, and let $T=\bigoplus_{n=1}^{\infty} T_{n}$. We have then, with $f=\sum_{n=1}^{\infty} f_{n}$, that

$$
\int \sup _{j}\left|f,(f+T f) / 2, \cdots,\left(f+\cdots+T^{j-1} f\right) / j\right|^{p(0)} d m=+\infty
$$

contradicting the hypothesis (a simple modification of the proof shows that we may restrict attention to a single measure space if it is nonatomic, by taking $\left(X_{n}, \mathscr{F}_{n}, \mu_{n}\right)=\left(X, \mathscr{F}_{,} \mu / 2^{n}\right)$, since $\bigoplus_{n=1}^{\infty}\left(X_{n}, \mathscr{F}_{n}, \mu_{n}\right)$ is then isomorphic $(X, \mathscr{F}, \mu)$ ).

THEOREM 3.3. Let $T$ be a positive contraction of $L_{p(0)}(X, \mathscr{F}, \mu)$, $1<p(0)<+\infty$, and let $f$ be a function in $L_{p(0)}(X, \mathscr{F}, \mu)$ such that $\|f\|=1$ and such that

$$
\int \sup _{j}\left|f,(f+T f) / 2, \cdots,\left(f+\cdots+T^{j} f\right) / j+1\right|^{p(0)}=+\infty .
$$

Then for each $k>0$, there exists a positive contraction $T(k)$ of $L_{p}(X, \mathscr{F}, \mu)$ such that

$$
\sup _{j} \mid g_{k},\left(g_{k}+T(k) g_{k} / 2, \cdots,\left(g_{k}+\cdots T^{j}(k) g_{k}\right) / j+\left.1\right|^{p(0)} \geqq k\right.
$$

for a function $g_{k}$ with $\left\|g_{k}\right\|=1$.

Proof. Let $M(f, T)=\sup _{j}\left|f, \cdots,\left(f+\cdots+T^{j} f\right) / j+1\right|^{p(0)}$. Since $\|M(f, T)\|=+\infty$, for each $k>0$ there exists a positive isometry $P$ of $L_{p(0)}(X, \mathscr{F}, \mu)$ such that $P(M(f, T)) \geqq k$. The lemma then follows if we define $T(k)=P T P^{-1}$ and $g_{k}=P f$, on noting that

$$
P(M(f, T))=\sup _{\jmath}\left|P f,(P f+P T f) / 2, \cdots,\left(P f+\cdots+P T^{\jmath} f\right) / j+1\right|^{p(0)} .
$$

This may be seen in the case that $(X, \mathscr{F}, \mu)$ is the denumerable union of Lebesgue spaces, since isometries of $L_{p(0)}(X, \mathscr{F}, \mu)$, in this case, admit of the following representation:

$$
P f=f(\tau x) \cdot r(x)
$$


where $\tau$ is an invertible measurable transformation, and where $r(x)$ is the nonnegative measurable function given by $r(x)=[d(\tau \mu) / d \mu]^{1 / p(0)}$ (see, e.g., [1], p. 5). In the general case, we may see the equality on noting that each $\sigma$-finite subspace is set isomorphic to a denumberable union of Lebesgue spaces.

THEOREM 3.4. If there exists a constant $c(p(0))$ such that

$$
T \in \mathscr{A}(p(0), p(0)), 1<p(0)<+\infty
$$

admits of a dominated estimate with constant $c(p(0))$ then the limit

$$
\lim (1 / n+1) \sum_{k=0}^{n} T^{k} f
$$

exists for each $f \in L_{p(0)}(X, \mathscr{F}, \mu)$.

Proof. The mean ergodic theorem for reflexive spaces gives us that

$$
L_{p}(X, \mathscr{F}, \mu)=\{\overline{f: f=g-T g}\}\{f: f=T F\} .
$$

The dominated estimate then shows that the limit exists provided that it exists for fixed functions, which is trivial, and provided it exists for functions of the form $g-T g$. In order to establish this fact, we need to show that $\lim _{n-\infty} T^{n} g / n=0$. This may be seen on noting that

$$
\int \sum_{n=1}^{\infty}\left|T^{n} f / n\right|^{p(0)} d \mu=\sum_{n=1}^{n} \int\left|T^{n} f / n\right|^{p(0)} d \mu<+\infty,
$$

as has been pointed out by M. A. Akcoglu.

\section{BIBLIOGRAPHY}

1. R. V. Chacon and J., Olsen, Dominated estimates of positive contractions, Proc. Amer. Math. Soc. 20 (1969), 266-271.

2. A. Ionescu Tulcea, Ergodic properties of isometries in $L_{p}$ spaces, $1<p<+\infty$, Bull. Amer. Math. Soc. 70 (1964), 366-371.

3. E. M. Stein On the maximal ergodic theorem, Proc. Nat. Acad. Sci. U. S. A. 47 (1961), 1894-1897.

Received January 17, 1969. Research supported in part by NSF grant GP-7490.

UNIVERSity OF MiNnESOTA 


\section{PACIFIC JOURNAL OF MATHEMATICS}

\section{EDITORS}

H. ROYDEN

Stanford University

Stanford, California

\section{RichaRd PIERCE}

University of Washington Seattle, Washington 98105
J. DugundJI

Department of Mathematics

University of Southern California

Los Angeles, California 90007

BASIL GORDON

University of California

Los Angeles, California 90024

\section{ASSOCIATE EDITORS}
E. F. BECKENBACH
B. H. NeUMANN
F. WOLF
K. YOSHIDA

\section{SUPPORTING INSTITUTIONS}

UNIVERSITY OF BRITISH COLUMBIA CALIFORNIA INSTITUTE OF TECHNOLOGY

UNIVERSITY OF CALIFORNIA

MONTANA STATE UNIVERSITY

UNIVERSITY OF NEVADA

NEW MEXICO STATE UNIVERSITY

OREGON STATE UNIVERSITY

UNIVERSITY OF OREGON

OSAKA UNIVERSITY

UNIVERSITY OF SOUTHERN CALIFORNIA

\author{
STANFORD UNIVERSITY \\ UNIVERSITY OF TOKYO \\ UNIVERSITY OF UTAH \\ WASHINGTON STATE UNIVERSITY \\ UNIVERSITY OF WASHINGTON \\ AMERICAN MATHEMATICAL SOCIETY \\ CHEVRON RESEARCH CORPORATION \\ TRW SYSTEMS \\ NAVAL WEAPONS CENTER
}

The Supporting Institutions listed above contribute to the cost of publication of this Journal, but they are not owners or publishers and have no responsibility for its content or policies.

Mathematical papers intended for publication in the Pacific Journal of Mathematics should be in typed form or offset-reproduced, double spaced with large margins. Underline Greek letters in red, German in green, and script in blue. The first paragraph or two must be capable of being used separately as a synopsis of the entire paper. It should not contain references to the bibliography. Manuscripts, in duplicate if possible, may be sent to any one of the four editors. Please classify according to the scheme of Math. Rev. 36, 1539-1546. All other communications to the editors should be addressed to the managing editor, Richard Arens, University of California, Los Angeles, California, 90024.

50 reprints are provided free for each article; additional copies may be obtained at cost in multiples of 50 .

The Pacific Journal of Mathematics is published monthly. Effective with Volume 16 the price per volume (3 numbers) is $\$ 8.00$; single issues, $\$ 3.00$. Special price for current issues to individual faculty members of supporting institutions and to individual members of the American Mathematical Society: $\$ 4.00$ per volume; single issues $\$ 1.50$. Back numbers are available.

Subscriptions, orders for back numbers, and changes of address should be sent to Pacific Journal of Mathematics, 103 Highland Boulevard, Berkeley, California, 94708.

PUBLISHED BY PACIFIC JOURNAL OF MATHEMATICS, A NON-PROFIT CORPORATION

Printed at Kokusai Bunken Insatsusha (International Academic Printing Co., Ltd.), 7-17. Fujimi 2-chome, Chiyoda-ku, Tokyo, Japan. 


\section{Pacific Journal of Mathematics}

\section{Vol. 30, No. $3 \quad$ November, 1969}

Willard Ellis Baxter, Topological rings with property $(Y) \ldots \ldots \ldots \ldots . \ldots 5$

Sterling K. Berberian, Note on some spectral inequalities of $C . R$.

Putnam ..................................... 573

David Theodore Brown, Galois theory for Banach algebras . ........... 577

Dennis K. Burke and R. A. Stoltenberg, A note on p-spaces and Moore spaces ........................................ 601

Rafael Van Severen Chacon and Stephen Allan McGrath, Estimates of positive contractions....................................... 609

Rene Felix Dennemeyer, Conjugate surfaces for multiple integral problems in the calculus of variations ........................... 621

Edwin O. Elliott, Measures on countable product spaces.............. 639

John Moss Grover, Covering groups of groups of Lie type .............. 645

Charles Lemuel Hagopian, Concerning semi-local-connectedness and cutting in nonlocally connected continua .................. 657

Velmer B. Headley, A monotonicity principle for eigenvalues ........... 663

John Joseph Hutchinson, Intrinsic extensions of rings . . . . . . . . . . . . . 669

Harold H. Johnson, Determination of hyperbolicity by partial

prolongations .................................. 679

Tilla Weinstein, Holomorphic quadratic differentials on surfaces in $E^{3} \ldots 697$

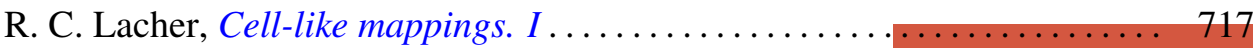

Roger McCann, A classification of centers

Curtis L. Outlaw, Mean value iteration of nonexpansive mappings in a

Banach space...

Allan C. Peterson, Distribution of zeros of solutions of a fourth order

differential equation.

Bhalchandra B. Phadke, Polyhedron inequality and strict convexity .. 765 Jack Wyndall Rogers Jr., On universal tree-like continua .

Edgar Andrews Rutter, Two characterizations of quasi-Frobenius rings

G. Sankaranarayanan and C. Suyambulingom, Some renewal theorems concerning a sequence of correlated random variables...

Joel E. Schneider, A note on the theory of primes........ . .

Richard Peter Stanley, Zero square rings .................

Edward D. Tymchatyn, The 2-cell as a partially ordered space

Craig A. Wood, On general Z.P.I.-rings................ 